\title{
Disruption of bone densitometry practice in a Northern Italy Orthopedic Hospital during the COVID-19 pandemic
}

\author{
C. Messina $^{1,2}$ - A. C. Buzzoni ${ }^{1} \cdot$ S. Gitto ${ }^{2} \cdot$ J. Almolla ${ }^{1} \cdot$ D. Albano ${ }^{1,3}\left(\mathbb{D} \cdot\right.$ L. M. Sconfienza $^{1,2}(\mathbb{D}$
}

Received: 29 May 2020 / Accepted: 4 August 2020 / Published online: 10 August 2020

(C) International Osteoporosis Foundation and National Osteoporosis Foundation 2020

\begin{abstract}
Summary

We report the impact of the COVID-19 pandemic on bone densitometry practice in a Northern Italy Orthopedic Hospital, comparing the first 4 months of 2020 with the corresponding period of 2019. COVID-19 pandemic had a disruptive effect on the daily practice of bone densitometry (about $-50 \%$ of examinations).

\section{Introduction}

The Coronavirus Disease 2019 (COVID-19) pandemic radically changes hospital organization to guarantee patient and staff safety, with the unavoidable cessation of normal outpatient activities. We report the impact of the COVID-19 pandemic on dual energy x-ray absorptiometry (DXA) testing in a Northern Italy Orthopedic Hospital.

\section{Methods}

We analyzed the number of DXA examinations performed at our Institution before, during the lockdown, and immediately after outpatient practice reopening (January 24th to May 27th, 2020), comparing them with the corresponding period of 2019.

\section{Results}

The number of DXA examinations showed a tremendous reduction from $n=1247$ performed from January to May 2019 to $n=$ 623 of $2020(-49.9 \%)$. No exams were performed in April $2020(-100 \%)$. On May 2020, a faint resume was observed, with $n=$ 43 DXA (-84.4\% compared to 2019$)$.

\section{Conclusion}

COVID-19 pandemic had a disruptive effect on the daily practice of bone densitometry with DXA. After reopening, we observed a persistence of DXA examination reduction, confirming the fact that returning to normality will probably be a slow process.
\end{abstract}

Keywords Dual energy X-ray absorptiometry $\cdot$ DXA $\cdot$ COVID-19 $\cdot$ Pandemic $\cdot$ Disruption $\cdot$ Bone

\section{Introduction}

The Coronavirus Disease 2019 (COVID-19) quickly spread worldwide from the region of Wuhan, China, and has been declared as a pandemic by World Health Organization on

C. Messina

carmelo.messina@unimi.it

1 IRCCS Istituto Ortopedico Galeazzi, Via Riccardo Galeazzi, 4, 20161 Milan, Italy

2 Dipartimento di Scienze Biomediche per la Salute, Università degli Studi di Milano, Via Pascal 36, 20100 Milan, Italy

3 Sezione di Scienze Radiologiche, Dipartimento di Biomedicina, Neuroscienze e Diagnostica Avanzata, Università degli Studi di Palermo, Via del Vespro 127, 90127 Palermo, Italy
March 11th, 2020 [1]. Italy was the first epicenter of the European outbreak, with the highest number of deaths up to May 4th $2020(28,884$ deaths, at that time second only to the United States) [2,3].

On February 20th, the first case of COVID-19 infection was reported in Italy [2]. Hence, the Italian Government established different measures to contain the spreading of virus, implementing distancing measures, finally leading to the national lockdown on March 9th [4]. Easing of restrictions (the so-called "phase 2") started from May 4th, and from May $18^{\text {th }}$, we further assisted to the reopening of many activities [5].

During this dramatic scenario, hospitals were forced to radically change their organization, in order to manage both COVID-19 and non-COVID-19 patients safely [6]. We assisted to an increased demand for intensive care unit beds, 
but hospitalization was required also from patients suffering from less severe COVID-19 forms [7]. This led to the cessation of normal outpatient activities with restricted access to hospitals $[4,6]$. Radiology departments were primarily involved by these measures, as they represent a crossroad of possible suspected COVID-19 subjects within hospitals [8].

Several medical societies provided recommendations to promote patient and staff safety during the pandemic, including the International Society for Clinical Densitometry (ISCD), which recognized that bone densitometry testing with dual energy x-ray absorptiometry (DXA) has been affected and idled by the crisis [9]. On March 30th, the ISCD Executive Committee stated that, during the emergency, any type of DXA examination should not be considered as essential to patients' health. Also, ISCD considered appropriate to delay or defer DXA examination for 3-6 months, as this would not affect patients' management [9].

The aim of this short communication is to report the impact of the COVID-19 pandemic on bone densitometry practice with DXA in the largest Italian Orthopedic Hospital, which is located in Milano, in the region which was first and most severely affected by the pandemic in Italy.

\section{Materials and methods}

\section{Overview of the local hospital situation during the COVID-19 outbreak}

The study was performed at IRCCS Istituto Ortopedico Galeazzi, which is an Orthopedic University and Research Hospital located in Milan, Lombardy (Italy). It is a private institution that mainly provides its services on behalf of the national public health system (Servizio Sanitario Nazionale, $\mathrm{SSN}$ ). In fact, the Italian SSN (which is regulated on a regional basis) relies on public financing, but services may be provided both by public and private institutions. Private hospitals may serve as SSN providers if they get an accreditation (a contract) with the specific region, therefore providing free of charge medical services as for public institutions [10].

Since the outbreak of infection (February 20th), all patients attending the hospital were asked to answer a questionnaire about suspected contacts with COVID-19 subjects and respiratory symptoms and had their temperature checked. The latter measure was soon extended to all employees. During the following weeks, the number of infected subjects quickly rose, and regional ordinance established specific roles for hospitals in Lombardy. After 3 weeks, dedicated floors and operating room were isolated and set up for COVID-19 patients, and the hospital was designed as regional hub for minor orthopedic trauma and selected orthopedic surgery (such as oncology or infectious disease). At this stage, the hospital spontaneously decided to suspend several elective activities, including non-urgent diagnostic imaging tests. On May 13th (about 1 week after the end of Italian lockdown), our radiology service reopened to regular outpatient activities, including DXA testing which are routinely performed 2 days a week. Of note, according to Italian regulations, DXA examinations are listed in the "essential levels of care" (livelli essenziali di assistenza, LEA), which means that these tests must be widely available to all Italian patients when clinical indications are fulfilled $[11,12]$.

\section{Data collection and analysis}

Data about DXA examinations performed at our Institution before, during the COVID-19 lockdown, and immediately after outpatient practice reopening (January 24th to May 27th, 2020) was collected from our information technology system. The same data regarding the corresponding period of 2019 was collected. Data was double-checked for accuracy with the internal database of our radiology department. For each timeframe, information about the type of DXA examination (lumbar spine, femoral, forearm, or whole body) was collected.

Numbers and sites of DXA examinations performed during the considered timeframe were compared with data obtained in the same timeframe of 2019. Data were analyzed using descriptive statistics for comparing the two corresponding timeframes using Microsoft Excel v. 16.0 (Microsoft Corporation, Redmond, WA).

\section{Results}

The number of DXA examinations showed a tremendous reduction from $n=1247$ performed between January and May 2019 to $n=623$ of the corresponding 2020 period ($49.9 \%$ ). An overview of the trend of DXA examinations performed during both timeframes is reported in Fig. 1.

More in detail, January and February 2019 and 2020 showed comparable numbers: in January 2019, we performed $n=229$ DXA versus $n=226$ of January 2020 ($1.3 \%$ ); in February 2019, we performed even less examinations $(n=239)$ compared with February $2020(n=279,+$ $16.7 \%$ ). The effect of COVID-19 outbreak started to be visible from March 2020, as we performed $n=75$ DXA compared with $n=281$ exams of the same period of 2019 ($73.3 \%$ ). No exams were performed in April 2020 ($100 \%$ ). On May 2020, we observed a faint resume of DXA testing, with $n=43$ DXA performed up to May 27th, a number that is sharply lower compared with May 2019 ($84.4 \%)$.

Regarding the specific distribution of DXA type during the two periods, on 2019, we performed DXA as follows: 645 lumbar spine DXA (51.7\%), 569 hip DXA (45.6\%), 21 
Fig. 1 Linear chart comparing the number of DXA examinations performed per during the first 4 months of 2019 and 2020. DXA = dual energy $\mathrm{x}$-ray absorptiometry
Total number of DXA examinations

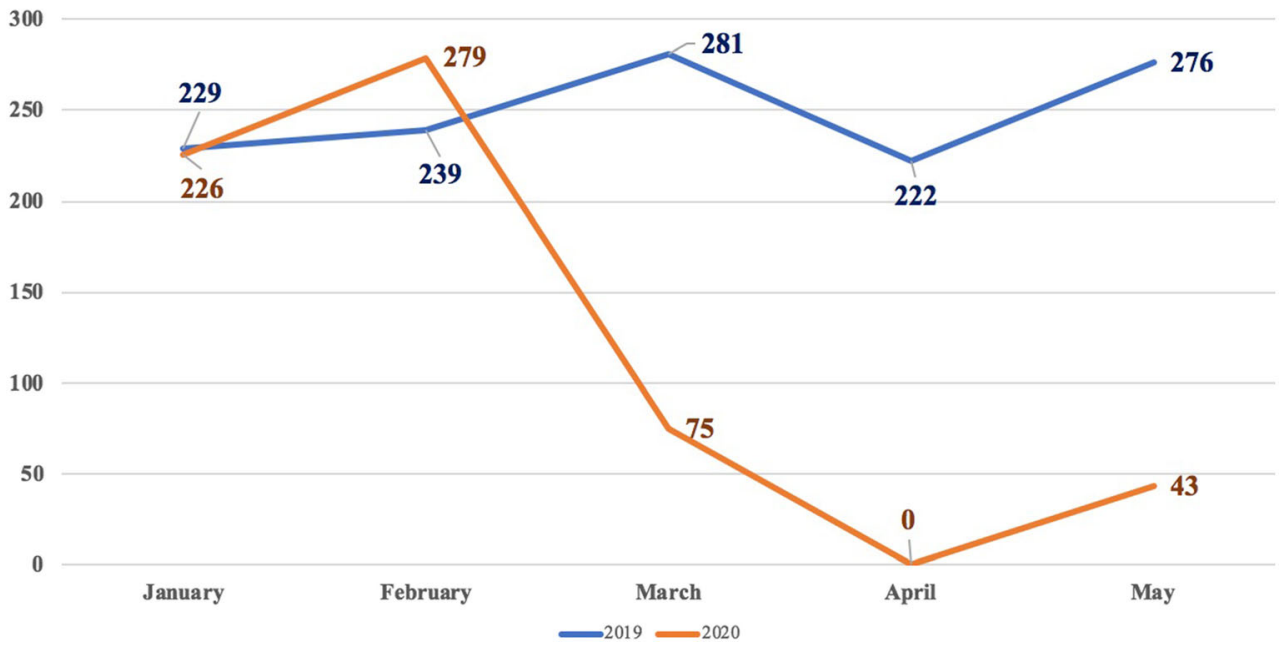

forearm DXA (1.7\%), 12 whole body DXA (1\%). During 2020, DXA were distributed as follows: 345 lumbar spine DXA (55.4\%), 267 hip DXA (42.9\%), 8 forearm DXA $(1.3 \%), 3$ whole body DXA $(0.5 \%)$ (Table 1$)$.

When comparing the mean age of the 43 patients examined after the "reopening" with those of the corresponding period of 2019, we found no statistically significant differences. In fact, patients in 2020 showed a mean age \pm standard deviation of $68.4 \pm 9.6$, while mean age of 2019 patients was $67.89 \pm$ $10.0(p>0.05)$.

\section{Discussion}

Our study reports the considerable impact of the COVID-19 outbreak on bone densitometry testing practice in a hospital located in one of the most affected Italian regions. After few weeks, it became immediately clear that a lockdown scenario was somehow inevitable for the majority of outpatient examinations for several reasons: first, the need to contain the infection and guarantee a safe environment both for patients and hospital staff, as the possibility of asymptomatic carriers quickly emerged [13]; second, the increasing number of infected persons became demanding for public health system,

Table 1 Distribution of number and types of DXA examination during the first 4 months of 2019 and 2020, with corresponding percentage values compared with total number of exams

\begin{tabular}{llrrrr}
\hline Year & Total & Lumbar spine & Hip & Forearm & Whole body \\
\hline 2019 & 1247 & 645 & 569 & 21 & 12 \\
& $(\%)$ & $52 \%$ & $46 \%$ & $2 \%$ & $1 \%$ \\
2020 & 623 & $34,500 \%$ & $26,700 \%$ & $800 \%$ & $300 \%$ \\
& $(\%)$ & $55 \%$ & $43 \%$ & $1 \%$ & $0 \%$ \\
\hline
\end{tabular}

with the need to preserve healthcare human and material resources (such as for example protective equipment). Moreover, we also assisted to spontaneous reduction of elective DXA examination even when the practice was still open, probably due to patients' concerns to undergo bone densitometry in this high-risk period.

As for many other radiological and non-radiological procedures, delaying medical services raised questions about the potential harmful consequences for patients' health [14]. The International Osteoporosis Foundation provided recommendations for older adults and people with osteoporosis during COVID-19 pandemic [15]. Similarly, the ISCD Executive Committee went out with a statement on March 30th to clarify that bone testing is not essential to individual's health and that a delay of 3-6 months is considered acceptable [9]. ISCD clearly stated that this 3-6-month restriction would not pose a threat to patients needing DXA scan, as for most of them, the BMD change is expected to be little and without clinical relevance [9]. Even in patients on osteoporotic treatment, or those who consider starting a treatment, the chance of a substantial change is considered very low. Additional information to this initial statement was provided on May 13th, due to the persistence of COVID-19 infection. The new statement was specifically aimed at preserving good densitometer function during the extended period of inactivity, by suggesting quality assurance procedures for each manufacturer brand [9].

In Italy, we already entered the phase of reduced restrictions, and while many business activities are reopening, public and private hospitals were allowed to slowly return to normality. This new phase of transition will probably be uncertain and will not resemble previous conditions of normality [14]. In fact, fear of infection may cause self-isolation of patients from hospitals and healthcare facilities, a situation that may further obstacle restoring the normal elective procedure rate. This was confirmed by our results, as during the first 2 weeks 
of reopening, we observed a considerable reduction in the number of DXA examinations compared with May 2019. This is partially related to the hospital's internal COVID-19 rules, which impose longer scheduled time for each patient in order to facilitate the social distancing between them.

Another problem that will surely create a delay to DXA testing will be the need for rescheduling postponed studies, a situation that may further contribute to stress hospital capacities both in terms of staff and machines.

A relevant aspect to be considered is that of possible pharmacologic treatment interruptions due to unwanted discontinuation of BMD testing caused by COVID-19 lockdown, as DXA remains the most commonly used technique for BMD evaluation [16]. It has been reported that BMD testing is associated to increased adherence to osteoporosis pharmacologic treatment, since its execution probably increase patients' awareness of osteoporosis risk, when the disease is diagnosed $[17,18]$. Future studies will certainly focus on this important topic when densitometric activity will return to pre-pandemic standards.

After the "reopening", we still had persistently low numbers of DXA measurement. In our opinion, this may be related to two main reasons. First, our hospital had to re-organize scheduling times due to the limitations imposed by social distancing, since we had to double the time between DXA examinations to reduce the number of patients in waiting rooms. Moreover, during the pandemic crisis, our hospital hosted a large number of COVID-19 patients. We believe that the fear of access to hospital that received COVID-19 patients may have influenced subjects in choosing to perform DXA examinations in smaller private centers where COVID-19 patients were not hospitalized.

We acknowledge that the retrospective design of our study represents a major limitation, but at the same time, we believe that another design would not have been possible, as Lombardy was one of the first and worst affected region of Italy, Europe, and Western countries.

In conclusion, we showed that COVID-19 pandemic had a disruptive effect on the daily practice of bone densitometry with DXA. After reopening of our bone densitometry service, we observed a persistence of DXA examination reduction, confirming the fact that returning to normality will probably be a slow process. Nevertheless, we believe that a delay of 36 months is an acceptable timeframe to contain the effect of COVID-19 disruption.

Data availability Data will be made available upon request.

\section{Compliance with ethical standards}

Conflicts of interest None.

Code availability Not applicable.

\section{References}

1. WHO. Coronavirus disease 2019 (COVID-19) Situation Report 51. https://www.who.int/docs/default-source/coronaviruse/ situation-reports/20200311-sitrep-51-covid-19.pdf?sfvrsn= 1ba62e57_10. Accessed 2 May 2020

2. Grasselli $\bar{G}$, Pesenti A, Cecconi M (2020) Critical care utilization for the COVID-19 outbreak in Lombardy, Italy. JAMA 323:1545. https://doi.org/10.1001/jama.2020.4031

3. WHO (2020) WHO. Coronavirus Disease 2019 (COVID-19): Situation Report - 105. WHO Coronavirus Dis 2019 Situat Rep $-10518$

4. Politi LS, Balzarini L (2020) The radiology department during the COVID-19 pandemic: a challenging, radical change. Eur Radiol 30:1-3. https://doi.org/10.1007/s00330-020-06871-0

5. Coronavirus: Italy's PM outlines lockdown easing measures - BBC News. https://www.bbc.com/news/amp/world-europe-52435273. Accessed 27 May 2020

6. Sorbello M, El-Boghdadly K, Di Giacinto I et al (2020) The Italian coronavirus disease 2019 outbreak: recommendations from clinical practice. Anaesthesia. 75:724-732. https://doi.org/10.1111/anae. 15049

7. D'Apolito R, Faraldi M, Ottaiano I, Zagra L (2020) Disruption of arthroplasty practice in an Orthopedic Center in Northern Italy during the coronavirus disease 2019 pandemic. J Arthroplast 35:S6-S9. https://doi.org/10.1016/j.arth.2020.04. 057

8. Mossa-Basha M, Meltzer CC, Kim DC, Tuite MJ, Kolli KP, Tan BS (2020) Radiology department preparedness for COVID-19: radiology scientific expert panel. Radiology 200988:E106-E112. https://doi.org/10.1148/radiol. 2020200988

9. ISCD COVID - 19 DXA Guidance - International Society for Clinical Densitometry (ISCD). https://www.iscd.org/about-iscd/ iscd-covid-19-dxa-guidance/. Accessed 27 May 2020

10. France G, Taroni F, Donatini A (2005) The Italian health-care system. Health Econ 14:S187-S202. https://doi.org/10.1002/ hec. 1035

11. Ministero della Salute Dipartimento della qualità (2005) Individuazione dei criteri di Accesso alla Densitometria Ossea

12. Rossini M, Adami S, Bertoldo F, Diacinti D, Gatti D, Giannini S, Giusti A, Malavolta N, Minisola S, Osella G, Pedrazzoni M, Sinigaglia L, Viapiana O, Isaia GC (2016) Guidelines for the diagnosis, prevention and management of osteoporosis. Reumatismo 68:1-39. https://doi.org/10.4081/reumatismo. 2016.870

13. Bandirali M, Sconfienza LM, Serra R, Brembilla R, Albano D, Pregliasco FE, Messina C (2020) Chest X-ray findings in asymptomatic and minimally symptomatic quarantined patients in Codogno, Italy. Radiology 295:201102. https://doi.org/10.1148/ radiol.2020201102

14. Luker GD, Boettcher AN (2020) Transitioning to a new normal after COVID-19: preparing to get back on track for cancer imaging. Radiol Imaging Cancer 2:e204011. https://doi.org/10.1148/rycan. 2020204011

15. COVID-19 and osteoporosis International Osteoporosis Foundation. https://www.iofbonehealth.org/news/covid-19-andosteoporosis. Accessed 27 May 2020

16. Messina C, Sconfienza LM, Bandirali M, Guglielmi G, Ulivieri F (2016) Adult dual-energy X-ray absorptiometry in clinical practice: how i report it. Semin Musculoskelet Radiol 20:246-253. https:// doi.org/10.1055/s-0036-1592370

17. Pressman A, Forsyth B, Ettinger B, Tosteson AN (2001) Initiation of osteoporosis treatment after bone mineral density testing. 
Osteoporos Int 12:337-342. https://doi.org/10.1007/ s001980170099

18. Rossi LMM, Copes RM, Dal Osto LC, Flores C, Comim FV,

Premaor MO (2018) Factors related with osteoporosis treatment in postmenopausal women. Medicine (Baltimore) 97:e11524. https://doi.org/10.1097/MD.0000000000011524
Publisher's note Springer Nature remains neutral with regard to jurisdictional claims in published maps and institutional affiliations. 